\title{
CÁLCULO DE LA DISPOSICIÓN A PAGAR POR LA CONSERVACIÓN Y MEJORA DE LOS SERVICIOS TURÍSTICOS DE LA LAGUNA DE PACUCHA
}

\author{
CALCULATION OF THE WILLINGNESS TO PAY FOR THE \\ CONSERVATION AND BY TO IMPROVING TOURIST SERVICES \\ PROVIDEDOF THE PACUCHA'S LAGOON
}

\author{
${ }^{1}$ Rolando Fredy Aguilar Salazar
}

\begin{abstract}
RESUMEN
La laguna de Pacucha y la vida silvestre que habita en su entorno constituyen un paisaje de gran belleza. Todos los años cientos de visitantes llegan a este lugar para disfrutar de la belleza del paisaje, de los animales y de los árboles y plantas. De no conservarse, se degradaría la belleza natural del área, se alteraría a los hábitats de los animales que aquí se protegen, se contaminaría el agua de la Laguna y por tanto disminuiría el número de visitantes; esto último podría también verse influido por otros factores como la calidad de los servicios turísticos que se brindan en los alrededores. El estudio tiene como objetivo determinar la disponibilidad de pago de los visitantes a la Laguna de Pacucha por su conservación, sus alrededores, y por la mejora de los servicios turísticos que se brindan en los alrededores. Para la estimación de la disposición a pagar se utilizó el Método de Valoración Contingente, el cual permite estimar el valor de bienes (productos o servicios) para los que no existe mercado. A través de la aplicación de 107 encuestas a visitantes a la Laguna de Pacucha, se determinó, con una probabilidad del 98,8\%, que los visitantes están dispuestos a pagar la cantidad de S/. 1,00 por cada visita. Del conjunto de ítems considerados en la encuesta sólo dos influyeron en la disponibilidad de pago a un nivel de confianza del $95 \%$.
\end{abstract}

Palabras clave: Valoración contingente, Disposición a pagar, Laguna de Pacucha.

\begin{abstract}
Pacucha's lagoon and wildlife inhabiting their environment constitute a landscape of great beauty. Every year hundreds of visitors come to this place to enjoy the beauty of the landscape, animals and trees and plants. If not preserved, natural beauty of the area is degraded, it would alter the habitats of the animals here are protected lagoon water would be contaminated and therefore decrease the number of visitors; at last could also be influenced by other factors such as the quality of tourism services provided nearby. The goal of this study is to determine the availability of paying visitors to the Pacucha's lagoon by conservation, its surroundings and by to improving tourist services provided nearby. To estimate the willingness to pay the Contingent Valuation Method, which allows to estimate the value of goods (products or services) for which there is no market was used. Through the application of 107 surveys of visitors to the Pacucha's lagoon was determined, with a probability of $98,8 \%$, which visitors are willing to pay the amount of S/. 1.00 for each visit. The set of items considered in the survey only two influenced the willingness to pay for a confidence level of $95 \%$.
\end{abstract}

Keywords: Contingent valuation, willingness to pay, Laguna Pacucha

\section{INTRODUCCIÓN}

La laguna de Pacucha es un área natural que se encuentra en el departamento de Apurímac - Perú y que alberga una gran variedad de plantas y animales propios de la zona. La laguna y la vida silvestre que habita en su entorno constituyen un paisaje de gran belleza. Todos los años cientos de visitantes llegan a este lugar para disfrutar de sus bon- dades. De no conservarse, se degradaría la belleza natural del área y se alteraría el hábitat de los animales. Por otro lado, de no mejorarse los servicios turísticos que se brindan en los alrededores, el número de visitantes disminuiría.

Según Valdivia et al. (2009) los servicios recreativos turísticos que ofrecen los ecosistemas, y que son parte de lo que se conoce como bienes ambientales, tienen como característica fundamental la de no tener exclusión en el consumo y

'Magister en Matemáticas, Licenciado en Matemáticas. Facultad de Ciencias de la Empresa de la Universidad Nacional José María Arguedas Andahuaylas-Perú. 
Aguilar, C. Cálculo de la disposición a pagar por la conservación y mejora de los servicios turísticos de la laguna de Pacucha.

de la falta de definición de los derechos de propiedad, lo que provoca que no exista, para ellos, un mercado en el cual se determine el precio y las cantidades demandadas y ofrecidas. Debido a lo anterior la oferta es insuficiente y en algunos casos nula, o bien los gastos en su mantenimiento están por debajo de los óptimos. Para conocer el precio potencial de estos bienes y servicios se utiliza, principalmente, el método de preferencias reveladas y el de preferencias declaradas o expresadas. En el de preferencias reveladas, el método específico y de mayor importancia reconocido por instituciones internacionales financiadoras de proyectos, programas y políticas ambientales, es el Método de Valoración Contingente.

El Método de Valoración Contingente consiste en formular un mercado hipotético mediante un cuestionario estructurado. Se trata, por tanto, de realizar una encuesta a una muestra representativa de la población por medio de la cual se ofrece una transacción no real entre el bien público a valorar y una cantidad monetaria. El objetivo del cuestionario es presentar un escenario creíble donde los individuos entrevistados constituyen la demanda y el entrevistador representa la oferta (Riera, 1994).

El Método de Valoración Contingente se basa en el supuesto económico de que las preferencias de los individuos pueden modelarse bajo una función de utilidad ordinal y donde dos estados de la naturaleza pueden ser interpretados y comparados en términos de los cambios en la función de utilidad (Hanneman, 1984).

El debate acerca de la validez del método se saldó con un informe favorable de la comisión de expertos de la Nacional Oceanic and Atmospheric Administration (NOAA), presidida por los premios Nobel de economía Kenneth Arrow y Robert Solow (Arrow, 1993). En su informe de enero de 1993 el panel de expertos se mostraba a favor del método como herramienta razonable para calcular el valor de no uso o uso pasivo en la pérdida de bienestar por desastres medioambientales. En este informe, además, recomendaban varias medidas bastante estrictas en su diseño y aplicación para evitar estimaciones exageradamente sesgadas que mencionamos a continuación:

1. La metodología de valoración contingente se debe aplicar por medio de entrevistas personales, antes que telefónicas.

Si no es posible la aplicación personal es preferible la realización de entrevistas telefónicas a las proporcionadas vía correo.

2. En los estudios de valoración contingente, se debe tratar de determinar la disposición a pagar (DAP) por un futuro accidente, antes que tratar de determinar la compensación mínima por un incidente que ya ocurrió.

3. En la aplicación de estos estudios se debe aplicar un formato de pregunta tipo Referéndum, el cual hace referencia a preguntas que solo tienen un SÍ y un NO como respuesta, en las cuales cada una de las respuestas individuales revela solamente un límite superior (para un No) o un límite inferior (para un $\mathrm{S}_{\mathrm{i}}$ ) de la medida de bienestar (Mendieta, 2001).

4. Los estudios de valoración contingente deben empezar mostrando un escenario que permita al lector entender los efectos del programa bajo consideración.

5. Se les debe recordar a los encuestados, que la DAP por una mejora en el bien ambiental en estudio les reducirá su renta futura.

6. En caso de la existencia de bienes sustitutos hay que hacerle saber al encuestado sobre su presencia. Por ejemplo, si a los encuestados se les está preguntando por una medida para regular o proteger algún área natural en particular, se les debería recordar que existen otras áreas naturales las cuales no necesitan la aplicación de estas medidas para seguir intactas.

7. Incluir preguntas de validación en la encuesta para verificar si hubo comprensión y aceptación del escenario planteado por parte del encuestado para detectar variables socioeconómicas y de actitud a incluir en la encuesta, lo cual permita un mejor análisis de los resultados.

El estudio realizado tiene como finalidad la conservación de la laguna, sus alrededores y la mejora de los servicios turísticos que se brindan en los alrededores; en ese sentido, el estudio tuvo como objetivo calcular el monto de disposición a pagar, en soles, de los visitantes a la laguna de Pacucha por su conservación, de sus alrededores y por la mejora de los servicios turísticos que se brindan en los alrededores. La justificación del monto puede justificar la realización de algún proyecto que posibilite dicha conservación y mejoras.

La viabilidad del monto que se plantea a los visitantes se determina teniendo en cuenta la formulación desarrollada por Hanemann (1984). Según esta formulación cada individuo posee su propia función de utilidad. Inicialmente, y en ausencia del bien ambiental, la utilidad personal será función del ingreso y de otras variables exógenas que miden su bienestar, nivel de educación, cobertura de salud, edad y recreación por ejemplo.

Esta relación está expresada por la ecuación:

$$
\mathrm{U}_{0}=\mathrm{f}(\mathrm{S})+\mathrm{bY}+\varepsilon_{0}
$$

Donde $\mathrm{U}_{0}$ es la utilidad sin algún proyecto de mejora del bien, Y el ingreso, S el vector de variables socioeconómicas, b la utilidad marginal del ingreso real y $\varepsilon_{0}$ un componente aleatorio (este componente se considera porque la función utilidad sin proyecto alguno de mejora no es observable en su totalidad).

Una vez que se realice algún proyecto y se cobren soles por él, la utilidad del individuo será $\mathrm{U}_{1}$. En ella el bienestar se ha alterado en y el ingreso se ha disminuido en, es decir:

$$
\mathrm{U}_{1}=\mathrm{f}(\mathrm{S}+\mathrm{dS})+\mathrm{b}(\mathrm{Y}+\mathrm{X})+\varepsilon_{1}
$$

Aquí $\mathrm{U}_{1}$ representa la utilidad con el proyecto y $\varepsilon_{1} \mathrm{el}$ componente aleatorio para la función utilidad con el proyecto.

Como es lógico, cada consumidor racional estaría dispuesto a pagar un valor $\mathrm{X}$ tal que su utilidad final resultante sea mayor que la inicial, y sea indiferente a pagar o no pagar si la utilidad permanece constante una vez realizado el proyecto. Este valor de equilibrio puede hallarse igualando (1) y (2):

$$
\begin{gathered}
\mathrm{f}(\mathrm{S})+\mathrm{bY}+\varepsilon_{0}=\mathrm{f}(\mathrm{S}+\mathrm{dS})+\mathrm{b}(\mathrm{Y}+\mathrm{X})+\varepsilon_{1} \\
X=\frac{f(S+d S)-f(S)+\varepsilon_{1}-\varepsilon_{0}}{b}
\end{gathered}
$$


Aguilar, C. Cálculo de la disposición a pagar por la conservación y mejora de los servicios turísticos de la laguna de Pacucha.

Para facilitar el estudio debe suponerse que la utilidad marginal (b) es constante; por tanto, el modelo deberá estimarse separadamente para personas con niveles de ingresos comparables.

En un formato de pregunta tipo referéndum, el individuo sólo responde "Sî" o "No", sin ser posible saber exactamente su disponibilidad a pagar. Es evidente entonces la necesidad de formular un modelo que permita estimar el valor máximo de que el individuo promedio de la muestra pagaría por el proyecto.

\section{Modelo de disponibilidad a pagar}

Suponiendo alguna distribución de probabilidades para el componente aleatorio $\varepsilon_{1}-\varepsilon_{2}$, puede estimarse una expresión para la probabilidad de que un individuo responda "Si" ante un valor escogido aleatoriamente para su encuesta.

La probabilidad de obtener un "Sî" por respuesta es igual a la probabilidad de que la utilidad final sea mayor que la inicial, es decir:

$$
\operatorname{Prob}(\mathrm{S} \hat{i})=\operatorname{Prob}\left(\mathrm{U}_{1}>\mathrm{U}_{0}\right)=\operatorname{Prob}\left[\varepsilon_{1}-\varepsilon_{0}<\mathrm{f}(\mathrm{S}+\mathrm{dS})-\mathrm{f}(\mathrm{S})-\mathrm{bX}\right]
$$

Si se conociera la distribución de probabilidades del componente $\varepsilon_{1}-\varepsilon_{0}$, entonces la igualdad previa podría escribirse explícitamente y despejar el valor de X.

$$
\begin{gathered}
\mathrm{Si} \varepsilon_{1}-\varepsilon_{0} \sim \mathrm{N}(0, \mathrm{k} 2)(\mathrm{k} \text { : constante), entonces: } \\
\operatorname{Prob}(S i)=\int_{-\infty}^{f(S+d S)-f(S)-b X} \frac{k}{k} \varphi(t) d t
\end{gathered}
$$

donde $\varphi(t)$ es la función de densidad de una normal con media cero y varianza 1 .

Si $\varepsilon_{1}-\varepsilon_{0}$ sigue una distribución logística con media cero y varianza 1 , entonces:

$$
\operatorname{Prob}(S i)=\int_{-\infty}^{\frac{f(S+d S)-f(S)-b X}{k}} \varphi(t) d t
$$

Debido a las formas que vemos en las igualdades previas la respuesta ante valores de $\mathrm{f}(\mathrm{S}+\mathrm{dS}), \mathrm{f}(\mathrm{S})$ y $\mathrm{X}$ varía entre 0 y 1 ; por tanto, puede estimarse un modelo, con base en la información muestral, entrando 1 cuando la respuesta ante un valor de sea "Si" y 0 cuando sea "No".

Para poder tener una forma explícita para $f(S+d S)-f(S)$ bX, Hanemann (1984) propone dos posibles formas funcionales:

\section{Forma lineal}

$$
\begin{gathered}
\mathrm{U}_{0}=\alpha_{0}+\mathrm{bY}+\varepsilon_{0} \\
\mathrm{U}_{1=} \alpha_{1}+\mathrm{b}(\mathrm{Y}-\mathrm{X})+\varepsilon_{1}
\end{gathered}
$$

donde $\alpha_{0}, \alpha_{1}$, b son funciones de S. En este caso $\mathrm{f}(\mathrm{S}+\mathrm{dS})-\mathrm{f}(\mathrm{S})-\mathrm{bX}$ queda descrita por:

$$
\alpha_{1}-\alpha_{0}-\mathrm{bX}
$$

En particular las ecuaciones (4) y (5) toman respectivamente las siguientes formas:

$$
\begin{aligned}
& \operatorname{Prob}(S i)=\int_{-\infty}^{\alpha_{1}-\alpha_{0}-b X} k^{k} \varphi(t) d t \\
& \operatorname{Prob}(S i)=\frac{1}{1+e^{-\left[\alpha_{1}-\alpha_{0}-b X\right]}}
\end{aligned}
$$

Forma semilogarítmica

$$
\begin{gathered}
\mathrm{U}_{0}=\alpha_{0}+\mathrm{b} \ln (\mathrm{Y})+\varepsilon_{0} \\
\mathrm{U}_{1=\alpha_{1}}+\mathrm{b} \ln (\mathrm{Y}-\mathrm{X})+\varepsilon_{1}
\end{gathered}
$$

donde $\alpha_{0}, \alpha_{1}$, b son funciones de S. En este caso $f(S+d S)-f(S)$-bX queda descrita por:

$$
\alpha_{1}-\alpha_{0}+\mathrm{b}[\ln (\mathrm{Y}-\mathrm{X})-\ln (\mathrm{Y})]=\alpha_{1}-\alpha_{0}+\mathrm{b} \ln (1-\mathrm{X} / \mathrm{Y})
$$

En particular las ecuaciones (4) y (5) toman respectivamente las siguientes formas:

$$
\begin{aligned}
& \operatorname{Prob}(S i)=\int_{-\infty}^{\alpha_{1}-\alpha_{0}-b \ln \left(1-\frac{X}{Y}\right)} \varphi(t) d t \\
& \operatorname{Prob}(S i)=\frac{1}{1+e^{-\left\{\alpha_{1}-\alpha_{0}-b \ln \left(1-\frac{X}{Y}\right)\right]}}
\end{aligned}
$$

\section{MATERIAL Y MÉTODOS}

\section{Material}

El estudio se llevó a cabo en los alrededores de la laguna de Pacucha del Distrito de Pacucha - Provincia de Andahuaylas - Departamento de Apurímac - Perú.

Para el procesamiento de datos se utilizó el software estadístico STATA versión 12.0.

\section{Métodos}

Para el logro del objetivo se empleó el Método de Valoración Contingente. El cuestionario que se elaboró tuvo las siguientes características:

A) Mercado hipotético: Representado por los bienes y servicios a valorar que se deben presentar sin ambigüedad a los encuestados, además de las amenazas. En este estudio ello se presentó como se muestra a continuación:

A1) Protección de animales y plantas del lugar: "La laguna de Pacucha es un área natural que alberga una gran variedad de plantas y animales propios de la zona. Todos los años cientos de personas, como usted, llegan a este lugar para disfrutar de la belleza del paisaje, de los animales y de los árboles y plantas" (texto incluido en el cuestionario).

A2) Belleza escénica: "La laguna y la vida silvestre que habita en su entorno constituyen un paisaje de gran belleza. Todos los años cientos de personas, como usted, llegan a este lugar para disfrutar de la belleza del paisaje, de los animales y de los árboles y plantas" (texto incluido en el cuestionario).

A3) Servicios turisticos: "Por otro lado, en este lugar, posiblemente usted haya hecho o hará uso de algún servicio turístico (el servicio de los restaurantes o tiendas por ejemplo)" (texto incluido en el cuestionario).

A4) Amenazas: "De no conservarse, se degradaría la belleza natural del área, se alteraría a los hábitats de los animales que aquí se protegen y se contaminaría el agua de la laguna". "De 
Aguilar, C. Cálculo de la disposición a pagar por la conservación y mejora de los servicios turísticos de la laguna de Pacucha.

implementarse más servicios turísticos y mejorarse los que ya existen seguramente usted se sentiría más a gusto" (textos incluidos en el cuestionario).

B) Vehículo de pago: Forma de cobrar una contribución que los visitantes estarían dispuestos a pagar por conservar el bien y mejorar los servicios turísticos. El mismo debe ser realista, conocido por los encuestados y estar relacionado con lo que se quiere valorar. En éste caso se optó por el pago de una entrada como mencionamos a continuación: "Tomando en cuenta lo anterior, ¿Estaría usted dispuesto a pagar la cantidad de 1 sol por cada visita que realice a este lugar para su conservación y la mejora de los servicios turísticos que se brindan?" (texto incluido en el cuestionario).

C) Tipo de pregunta: Teniendo en cuenta las recomendaciones del panel NOAA se optó por la Disposición a Pagar.

D) Formato de pregunta: Se utilizó el formato Referéndum.

E) Estructura del cuestionario: La encuesta empleada para extraer la disposición a pagar constó de tres bloques. El primer bloque contenía información referida al servicio ambiental y turístico, esto asegura que el encuestado conozca el problema que se está tratando. El segundo bloque describía la modificación de los servicios ambientales y turísticos, los resultados positivos que se lograrían y la disposición de pago. El tercer bloque recogía información sobre las características socioeconómicas más relevantes del encuestado.

F) Administración del cuestionario: Se eligió la encuesta personal y se aplicó en el mismo lugar.

G) Estudio piloto: Siguiendo las recomendaciones de Arrow (1993), para evaluar la comprensión de las preguntas por parte de las personas encuestadas ( $\mathrm{y}$ así la validez interna del método), el tiempo destinado a responder el cuestionario, definir los ítems a incluir y como parte del entrenamiento del encuestador, se elaboró un cuestionario piloto. El mismo se administró a 30 personas que estaban haciendo turismo en el lugar. Este cuestionario contenía una serie de preguntas preliminares divididas en tres bloques según lo antes mencionado, que luego se ajustó en función de los resultados obtenidos. Junto con las respuestas se recibió observaciones y aportes de parte de los encuestados. El estudio piloto se aplicó durante los meses de setiembre, octubre y noviembre de 2012.

H) Determinación del tamaño muestral: En el estudio, se aplicaron 126 encuestas. Este número fue obtenido utilizando la fórmula:

$$
n=\frac{\left(Z_{1-\frac{\alpha}{2}}\right)^{2} p q}{e^{2}}
$$

donde $\alpha$ se fijó en $5 \%$, en $7 \%$ y fue calculado en la encuesta piloto dando el valor de 0,8 . De este número de encuestas se descartaron 19 encuestas porque los encuestados solo respondieron parte de la encuesta o marcaban más de una alternativa en las encuestas.

I) Estudio principal: El cuestionario final fue administrado a 107 personas que estaban haciendo turismo en el lugar durante los meses de enero a agosto de 2013. Se encuestó a los jefes de los grupos que se encontraban en el lugar, mayores de 18 años, asumiendo que éstos tienen una idea del valor real del dinero.

La viabilidad del monto planteado en el cuestionario depende del valor de la probabilidad de estar dispuesto a pagar; en ese sentido, se utilizó, teniendo en cuenta la propuesta hecha por Hanemann (1984), el modelo representado por la ecuación (5) en su forma lineal, se utilizó el modelo:

Tabla 1. Descripción de las variables para la Disposición a pagar

\begin{tabular}{|c|c|c|}
\hline Variable: & Descripción & Valores que toma \\
\hline var1 & Número de personas menores de 18 años con las que vino & $1=$ Con ninguna; $2=1$ ó $2 ; 3=3$ o más \\
\hline var2 & Lugar donde reside & $\begin{array}{l}\text { 1=En uno de los siguientes distritos: San Jerónimo, Andahuaylas, Talavera; } 2= \\
\text { El otro distrito, pero dentro de la provincia de Andahuaylas; } 3=\text { Fuera de la } \\
\text { provincia de Andahuaylas }\end{array}$ \\
\hline var3 & Número de visitas anuales que realiza al lugar & $1=$ De 1 a 2 visitas; $2=$ De 3 a 5 visitas; $3=$ De 6 a más visitas \\
\hline var4 & Motivos por el que ha visitado el lugar & $\begin{array}{l}1=\text { Disfrutar del paisaje y } / o \text { aire puro; } 2=\text { Observar los animales, plantas y } \\
\text { animales; } 3=\text { Otros motivos }\end{array}$ \\
\hline var5 & $\begin{array}{l}\text { Sobre si solicitó o no la atención y/o servicio de algún } \\
\text { restaurante y/o tienda del lugar }\end{array}$ & $1=\mathrm{Si}, 1=\mathrm{No}$ \\
\hline var6 & $\begin{array}{l}\text { Tipo de atención y/o servicio que brindan los restaurantes } \\
\text { y/o tiendas }\end{array}$ & $1=$ Malo, $2=$ Regular, $3=$ Bueno \\
\hline var7 & Sobre implementación de otros servicios turísticos & $1=\mathrm{Si}, 2=$ No sabe $/$ No opina, $3=\mathrm{No}$ \\
\hline $\operatorname{var} 8$ & Disposición a pagar & $1=\mathrm{Si}, 0=\mathrm{No}$ \\
\hline var9 & Razones por la no disposición a pagar & $1=$ Razones económicas, $2=\mathrm{El}$ gobierno debería pagar, $3=$ Otros \\
\hline $\operatorname{var} 10$ & Institución apropiada para recepcionar el pago & $\begin{array}{l}1=\text { El Ministerio de Comercio Exterior Turismo; } 2=\mathrm{La} \text { alcaldia de Pacucha; } \\
3=\text { Otros; }\end{array}$ \\
\hline var11 & Sexo del entrevistado & $1=$ Masculino, $2=$ Femenino \\
\hline $\operatorname{var} 12$ & Edad del entrevistado & Números enteros $>=18$ \\
\hline var12M & Edad del entrevistado (categorizado) & $1=$ Entre 18 y 40 años, $2=$ Entre 41 y 60 años, $3=$ Mayor a 60 años \\
\hline $\operatorname{var} 13$ & Nivel de educación del entrevistado & $1=$ Primaria, $2=$ Secundaria, $3=$ Superior más \\
\hline var14 & Rango más cercano al ingreso económico familiar mensual & $1=1000$ soles o menos, $2=$ Entre 1001 y 2000 soles, $3=$ Más de 2000 soles \\
\hline
\end{tabular}


Aguilar, C. Cálculo de la disposición a pagar por la conservación y mejora de los servicios turísticos de la laguna de Pacucha.

$$
\operatorname{Prob}(S i)=\frac{1}{1+e^{-\left[\alpha_{1}-\alpha_{0}-b X\right]}}
$$

Para determinar las variables que entran en este modelo se utilizó la técnica estadística de regresión logística binaria (Dominguez y Aldana, 2001 y Sanchez, 2000), pues se adecua perfectamente al mismo.

A través del software STATA 12.0 , los datos se ajustaron al modelo de regresión logística binaria "logit", siendo la Disposición a Pagar la variable dependiente. Este método estadístico cuantifica cómo influye en la probabilidad de aparición de un suceso dicotómico (en este caso estar o no dispuesto a pagar el precio propuesto), ciertos factores que se presumen relevantes o influyentes, y es la forma estadísticamente correcta de modelar respuestas de tipo dicotómicas (Michael y Carson, 2005; Loomies et al., 2009, citados por Zappi, 2011). Para esto, se ingresaron los datos de cada una de las encuestas en una matriz, con las siguientes especificaciones dadas en la tabla 1:

\section{RESULTADOS}

En la tabla 2 y figura 1, se presenta los resultados sobre la disposición de pago de los visitantes a la Laguna de Pacucha.

Tabla 2. Disponibilidad a pagar (DAP)

\begin{tabular}{cccc}
\hline & Frecuencia absoluta & Porcentaje & Porcentaje acumulado \\
\hline $\mathrm{Si}$ & 83 & 77,57 & 77,57 \\
No & 24 & 22,43 & 100,00 \\
Total & 107 & 100,00 & \\
\hline
\end{tabular}

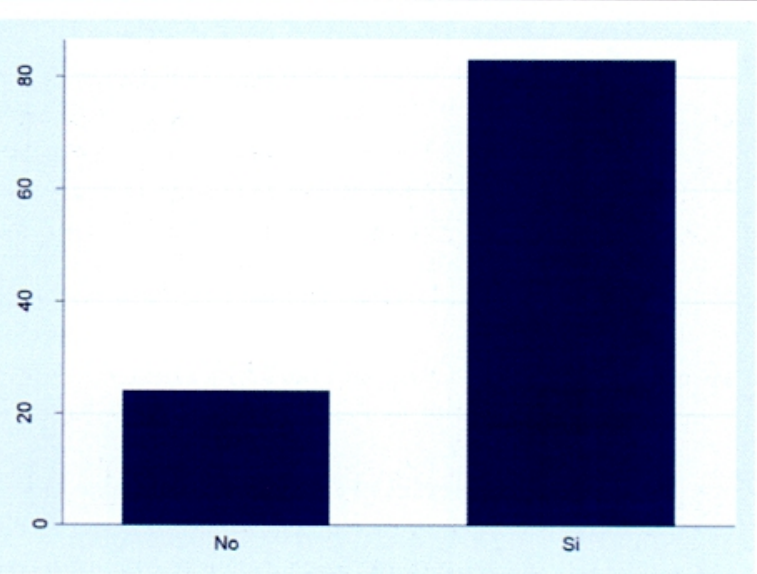

Figura 1. Disponibilidad a pagar (DAP) la cantidad de $1 \mathrm{sol}$
En la tabla 3 se presenta los resultados de la regresión exploratoria, en que cada variable se corrió individualmente contra la Disposición a Pagar para tener una idea sobre que variables incluir en el modelo final.

En la tabla 4 se presenta los resultados de la regresión exploratoria, en que las variables seleccionadas de la tabla anterior $\left(^{*}\right)$ se corrieron en conjunto contra la Disposición a Pagar para ver que variables incluir en el modelo final.

En la tabla 5 se presenta los resultados de la regresión exploratoria, en que las variables seleccionadas de la tabla anterior se corrieron en conjunto contra la Disposición a pagar para obtener el modelo final.

Teniendo en cuenta las tablas 1 y 5 , el modelo de la $\mathrm{P}($ Disposición a Pagar=1) sería el siguiente:

$$
=\frac{1}{1+e^{-[-10,84107+3,772613 \operatorname{Var} 13+3,242842 \operatorname{Var} 14]}}
$$

$\mathrm{Al}$ introducir en esta ecuación los valores promedio de la muestra para cada uno de los factores se obtiene que la probabilidad de estar dispuesto a pagar 1 sol por la conservación de la Laguna de Pacucha, sus alrededores y la mejora de los servicios turístico es 0,988 .

$$
=\frac{1}{1+e^{-[-10,84107+3,772613(2,345794)+3,242842(1,962617)]}}=0,988
$$

Tabla 4. Coeficiente y Significancia de las variables seleccionadas como variables que explican la Disposición a Pagar

\begin{tabular}{lc}
\hline Variables & Coeficien Significanc \\
\hline Motivos por el que ha visitado el lugar & $-0,39843130,511$ \\
Sobre implementación de otros servicios & $-0,09934870,923$ \\
turísticos & $-1,2898 \quad 0,142$ \\
Edad del entrevistado & $2,8219340,048^{*}$ \\
Nivel de educación del entrevistado & $3,7068990,009 *$ \\
Rango más cercano al ingreso económico & \\
familiar mensual & \\
${ }^{*} \mathrm{p}<0,05$ &
\end{tabular}

Tabla 5. Coeficiente y Significancia de las variables seleccionadas finales como variables que explican la Disposición a pagar.

\begin{tabular}{|c|c|c|}
\hline Variables & Coeficiente & Significancia \\
\hline Número de personas menores de 18 años con las que vino & $-0,2673396$ & 0,398 \\
\hline Lugar donde reside & 0,508708 & 0,399 \\
\hline Número de visitas anuales que realiza al lugar & 0,4705412 & 0,309 \\
\hline Motivos por el que ha visitado el lugar & $-1,068504$ & $0,004^{*}$ \\
\hline Sobre si solicitó o no la atención y/o servicio de algún restaurante y/o tienda del lugar & $-0,2600933$ & 0,615 \\
\hline Tipo de atención y/o servicio que brindan los restaurantes y/o tiendas & $-0,2773062$ & 0,641 \\
\hline Sobre implementación de otros servicios turísticos & $-3,859282$ & $0,000^{*}$ \\
\hline Sexo del entrevistado & 1,019148 & 0,064 \\
\hline Edad del entrevistado & $-1,259354$ & $0,001^{*}$ \\
\hline Nivel de educación del entrevistado, & 4,043704 & $0,000^{*}$ \\
\hline Rango más cercano al ingreso económico familiar mensua! & 3,930587 & $0,000^{*}$ \\
\hline
\end{tabular}

\begin{tabular}{lcc}
\hline Variables & Coeficiente & Significancia \\
\hline Nivel de educación del entrevistado & 3,772613 & $0,005^{*}$ \\
Rango más cercano al ingreso económico & 3,242842 & $0,003^{*}$ \\
Constante & $-10,84107$ & $0,001^{*}$ \\
\hline${ }^{*}<00,05$ & &
\end{tabular}

Tabla 3. Coeficiente y Significancia de cada variable como variables que explican la Disposición a Pagar ${ }^{*} \mathrm{p}<0,05$ 
Aguilar, C. Cálculo de la disposición a pagar por la conservación y mejora de los servicios turísticos de la laguna de Pacucha.

\section{DISCUSIÓN}

Según la tabla 2, el 77,57\% de los encuestados tiene la disposición de pagar 1 sol y el restante $22,43 \%$ no. Esto significa que casi todos los encuestados pudieron manifestar una disposición a pagar, afirmativa o negativa. Se interpreta entonces que la mayoría de las personas fueron capaces de realizar un análisis costo-beneficio para responder sobre su disposición a pagar, es decir que pudieron evaluar las consecuencias económicas de su decisión. Esto también refleja que la valoración contingente pudo ser conducida exitosamente en los alrededores de la Laguna de Pacucha, y que el mercado hipotético planteado fue creíble y comprensible para los encuestados.

El modelo final obtenido, luego de las corridas con el software, indica que sólo dos variables explican en conjunto la disposición de pago.

La probabilidad de la disposición a pagar por los visitantes, representada por el $98,8 \%$ proporciona la confianza necesaria de la aplicación del cuestionario.

\section{CONCLUSIONES}

Luego de aplicar el Método de Valoración Contingente a los visitantes a la Laguna de Pacucha, se puede concluir que, por primera vez, existen elementos de base involucrados en el proceso de valoración de los sistemas naturales y sus componentes que existen en ese lugar.

Los resultados de las encuestas revelan que el $77,57 \%$ de los encuestados está dispuesto a pagar la cantidad de S/. 1.00 por persona para la conservación de la Laguna de Pacucha, sus alrededores y la mejora de los servicios turísticos que se brindan en los alrededores.

Las encuestas piloto que determina el monto de disposición a pagar son muy importantes.

La utilización del Método de Valoración Contingente es importante, pues proporciona el modelo del cuestionario y el modelo para el cálculo de la $\mathrm{P}$ (Disposición a pagar=1).

La técnica estadística de regresión logística binaria constituye una herramienta importante para determinar las variables que intervienen en el modelo final de la disposición a pagar de los visitantes. .

\section{REFERENCIAS BIBLIOGRÁFICAS}

Arrow, K., Solow, R., Portney, P., Leamer, E., Radmer, R., and Schuman, H. (1993). Report of the NOAA
Panel on Contingent Valuation, Federal Register, Washington DC, January 15.

Azqueta, D. (1994), Valoración Económica de la Calidad Ambiental. Madrid, España: Ed. Alianza.

Cristeche E., Penna J. (2008). Métodos de Valoración Económica de los Servicios Ambientales. Editorial INTA, ISNN $1851-6955, \mathrm{~N}^{\circ} 3,58$ pág.

Dominguez E. y Aldana D. (2001), Regresión logística. Un ejemplo de su uso en endocrinología, Revista Cubana Endocrino, $\mathrm{N}^{\circ} 12$,pp 58-64.

Hanneman, W. (1984). Welfare evaluations in contingent valuation experiments with discrete responses, American Journal of Agricultural Economics, Nº6 (3), pág. 332-341.

Labandeira X., León C., Vasquez M. (2007). Economía Ambiental, Madrid, España: Editorial Pearson, 376 pág.

Mendieta, J. C. (2001). Manual de valoración económica de bienes no mercadeables, Universidad de los Andes, Facultad de economía, pág 91-117.

Riera, P. (1994). Manual de Valoración Contingente. Instituto de Estudios Fiscales.

Sánchez E. (2000). Regresión logistica en Salud Pública. España: Editorial Escuela Andaluza de Salud Pública., 173 pág.

Sánchez J. (2008). Valoración Contingente y Costo de Viaje aplicados al área recreativa laguna de Macubaji, RevistaEconomia, Vol. XXXIII, pág. 119-150.

Sanjurjo E. \& Islas I. (2007). Valoración económica de la actividad recreativa en el Río Colorado (Estados Unidos y México). Revista Región y Sociedad, vol XIX, $\mathrm{N}^{\circ} 40,26$ pág.

Rivas A. y Ramoni J. (2007). Valoración Contingente aplicada al caso del Río Albarregas Mérida-Venezuela, Revista Fermentum Vol. 17, $\mathrm{N}^{\circ}$ 49, pp. 478-502, 26 pág.

Valdivia R., Cuevas C., Sandoval M. y Romo J. (2009). Estimación econométrica de la disponibilidad a pagar por los consumidores de servicios recreativos turísticos, Revista Terra Latinoamericana, Vol. 27, Núm. 3, pp. 227-335.

Vásquez F., Cerda A. y Orrego S. (2007). Valoración económica del ambiente, 1 ra edición, Argentina, Editorial Thomson, 365 pág.

Zappi L. M. (2011). Valoración Contingente: Explorando la disposición a pagar por servicios ambientales declarada por usuarios de la reserva nacional Lago Peñuelas (Tesis de pregrado), Chile, Universidad de Chile.
Correspondencia:

Rolando Fredy Aguilar Salazar: r-aguilar-s@hotmail.com
Fecha de Recepción: 08/05/2015

Fecha de Aceptación: 20/10/2015 